\title{
Management of Severe Dry Eye: Role of Autologous Serum Eye Drops
}

\author{
Achyut N Pandey \\ Department of ophthalmology, VCSG medical college and research institute, srikot, Srinagar garhwal, uttarakhand, INDIA
}

Received: 15 July, 2017; Accepted: 30 September, 2017; Published: 27 October, 2017

*Corresponding author: Achyut N Pandey, Department of ophthalmology, VCSG medical college and research institute, srikot, Srinagar garhwal, uttarakhand-246174, India; E-mail:achyutpandey@gmail.com

\begin{abstract}
Theoretically, autologous serum eye drops have a potential advantage over traditional therapies based on the assumption that AS serve not only as a lacrimal substitute to provide lubrication, but also contain other biochemical components mimicking natural tears more closely. The application of AS in dry eye treatment has gained popularity as a second-line therapy in the treatment of dry eye. Published studies on the subject indicate that autologous serum could be an effective treatment for dry eye. Tear film plays an important part in the maintenance of intact ocular surface with their anti-microbial, nourishing, mechanical and optical properties. Components of tears include growth factors, fibronectin and vitamins to support proliferation, migration and differentiation of the corneal and conjunctival epithelium. Depletion in quantity or quality of tears can lead to various changes like squamous metaplasia, epithelial defects and infections of corneal epithelium. Artificial tears used in treatment of dry eye provide lubrication to the ocular surface, but they fall short of functioning as tears due to lack of nutrients and factors present in natural tears. Autologous serum drops being prepared from patient's body fluids are thus non-allergic and their biomechanical and biochemical properties are similar to normal tears. Autologous serum eye drops can provide lubrication and important biochemical components that mimic natural tears for dry eye patients. Serum eye drops are becoming a more popular second-line therapy to treat dry eye. This study explains the role of Autologous serum drops in dry eye [1-3].
\end{abstract}

\section{Introduction [4-6]}

The eye depends on the presence of a tear film to provide constant moisture and lubrication to maintain vision and comfort. Tears are a combination of: water, for moisture; oils, for lubrication and to prevent evaporation of tear liquid; mucus, for even spreading of tears on the surface of the eye; and, antibodies and special proteins, for resistance to infection.

These components are secreted by special glands located around the eye. When there is an imbalance or deficiency in this tear system, or when the tears evaporate too quickly, a person may experience dry eye. Pain, burning, a gritty sensation, like a feeling of a foreign body or sand, itching, redness and blurring of vision. Sometimes, a person with dry eye will have excess tears running down the cheeks, which may seem confusing. This happens when the eye isn't getting enough lubrication. The eye sends a distress signal through the nervous system for more lubrication. In response, the eye is flooded with emergency tears [7-10].

\section{Discussion}

Dry eye is a common disorder, with an estimated $25 \%$ of patients in general ophthalmology or optometry clinics reporting dry eye symptoms. It is known that the incidence of dry eye increases with age and has a higher prevalence in women compared to men [8-10]. Recently, the Definition and Classification Subcommittee of the International Dry Eye Work Shop, redefined dry eye as "a multifactorial disease of the tears and ocular surface that results in symptoms of discomfort (including foreign body sensation, dryness or irritation, burning, light sensitivity, redness), visual disturbance, secretion with crusting on the eyelashes, and tear film instability with potential damage to the ocular surface. It is accompanied by increased osmolarity of the tear film and inflammation of the ocular surface". An increased tear osmolarity, which causes ocular surface inflammation, is thought to be the central pathogenic mechanism of dry eye. The mechanistic classification of dry eye suggested by the DEWS defines two main subtypes: aqueous deficiency and evaporative dry eye, respectively corresponding to disorders of the lacrimal and meibomian glands. Disorders of the lacrimal and meibomian glands are usually secondary to either systemic diseases or local causes. One of the most common systemic diseases causing dry eye is Sjögren's syndrome. It presents as "sicca complex," a combination of dry eye and dry mouth (xerostomia) due to a T-lymphocyte mediated destruction of the exocrine glands. Other systemic diseases, such as rheumatoid arthritis, diabetes, systemic lupus erythematosus, dermatological conditions such as acne rosacea, and Graves' disease have also been reported as causing clinically significant dry eye. On the other hand, the leading causes for non-systemic disease-related dry eye include agerelated lacrimal dysfunction, hormonal changes, drug side-effects (e.g. systemic antihistamines, diuretics, topical beta blockers for glaucoma therapies), surgical intervention (e.g. photorefractive keratectomy and laser in situ keratomileusis, as well as longterm contact lens use $[10,11]$. The diagnosis of dry eye is made by 
validated patient symptom questionnaires and with a wide array of clinical assessments of the tears and ocular surface. Symptoms of dry eye have been standardized by use of questionnaires. The most common complaints described by patients include dryness or irritation, light sensitivity, foreign body sensation, red eyes, poor vision, daily life limitations, and symptom fluctuation in different environmental conditions. However, it has also been noted that there is no strong correlation between signs and symptoms, particularly in mild dry eye. Therefore, the clinical diagnosis of dry eye needs to incorporate objective tests such as tear osmolarity, tear production by Schirmer's testing, fluorescein clearance, fluorescein break-up time, and demonstration of ocular surface damage through dye staining (fluorescein and lissamine green). Although there is presently no gold standard diagnostic test to identify dry eye, a growing number of studies have suggested that tear osmolarity might be the best single metric for diagnosis and severity assessment of dry eye [12-14].

Currently there is no cure for dry eye. Common treatments are targeted to manage the symptoms. The mainstay of conventional therapy is the application of artificial tears that increase moisture on the ocular surface, and provide additional lubrication. A variety of artificial tear formulations differ from each other in their electrolyte composition, osmolarity, viscosity, the presence of preservatives, and compatible solutes. An unpreserved artificial tear containing $0.1 \%$ sodium hyaluronate was found to be effective in improving dry eye symptoms with a significant improvement in the mean tear film osmolarity, break-up times, and conjunctival and corneal staining scores. However, the use of artificial tears has some limitations. Natural tears have a complex composition of water, salts, hydrocarbons, proteins, and lipids that artificial tears cannot exactly substitute. Additionally, frequent application of artificial tears solutions containing chemical preservatives to prevent contamination has been found to induce toxic and allergic reactions, especially among those with sensitive eyes $[14,15]$.

Topical corticosteroids that target the inflammatory pathways associated with ocular inflammation have been shown to improve symptoms in people with dry eye, but their use is limited due to long-term side effects including cataracts and increased intraocular pressure. In December 2002, the U.S. Food and Drug Administration approved $0.05 \%$ solution of cyclosporine A as an ocular therapeutic for people with dry eye. Several studies have shown an increase in tear production and conjunctival goblet cell density with few reported adverse effects following the topical application of CsA .

Additional nutritional supplements such as essential fatty acids, including omega-3, linoleic acid, and gamma-linoleic acid, have been proposed as adjuvants in the treatment of dry eye due to their anti-inflammatory properties. Increased water intake and reduced alcohol consumption are also recommended to improve dry eye symptoms. Environmental interventions designed to increase air moisture and reduce particles in the air, including indoor humidifiers and air filters or cleaners have been shown to reduce dry eye symptoms as well. For people in whom artificial tears are not sufficient, preservation of the tear film can be achieved by inserting punctal plugs in the lacrimal ducts, designed to reduce the drainage of tears through the lacrimal ducts and increase lubrication on the ocular surface [15].

\section{Description of the intervention}

The composition of serum resembles that of tears; most concentrations are equivalent, with the exception of more vitamin A, lysozyme, transforming growth factor- $\beta$ (TGF- $\beta$ ) and fibronectin, and less immunoglobulin A (IgA), epithelial growth factor (EGF) and vitamin C in serum than in tears. Since many of the essential components in tears are present in serum, the use of serumas a tear substitute for the maintenance of the ocular surface seems feasible. In 1975, autologous serum eye drops were initially applied for dry eye. Since then, AS have become increasingly popular for treating ocular surface diseases, mainly dry eye.

Preservatives are usually not added to AS, thus reducing the risk of preservative-induced toxicity associated with other dry eye treatments. However, the lack of preservatives theoretically increases the risk of ocular infections. Autolgous serum can be stored for less than one month at $4{ }^{\circ} \mathrm{C}$ while in use, and for up to three months at $-20^{\circ} \mathrm{C}[15,16]$.

\section{Indications}

Autologous serum eye drops have been recommended for the treatment of several ocular surface disturbances, such as Sjögren's syndrome-related tear deficiency, non-Sjögren's tear deficiency associated with graft-versus-host disease, neurotrophic keratitis, persistent epithelial defects, superior limbic keratoconjunctivitis, and postoperative dry eye induced by LASIK. Serum contains various factors that are also present in tears including vitamin A, epidermal growth factors, substance $\mathrm{P}$, as well as proteins such as lactoferrins and lysozymes. All these factors are essential for healthy functioning of ocular surface. Biochemical properties of normal human tears and serum are approximately similar with vitamin A, fibronectin, transforming growth factor beta been more in serum and surface immunoglobulin a more in tears. Also, there is no difference between growth factor levels of autologous serum of dry eye syndrome and normal person. Transforming growth factor beta is also believed to control epithelial proliferation and to maintain cells in undifferentiated state. Neural factors such as substance $P$ are important for corneal epithelial migration. Human serum stimulates corneal fibroblast migration, proliferation and matrix metalloproteinase activity. Presence of oil in serum may act as a replacement for lipid tear component produced by the meibomian glands. Impression cytology studies also suggest that autologous serum leads to up regulation of goblet cells and mucin expression in a dose dependent manner. Autologous serum eye drops improve conjunctival status too in dry eye syndrome patients. Autologous serum eye drops were found effective and safe in treatment of severe dry eye disease, as evidenced by improvement in subjective assessment of symptoms, Schirmer's score, tear film break-up time and ocular surface staining [16-18]. 


\section{Conclusion}

Autologous serum eyedrops were found effective and safe in treatment of severe dry eye disease, as evidenced by improvement in subjective assessment of symptoms, Schirmer's score, tear film break-up time and ocular surface staining.

\section{References}

1. Albegger KW, Tilz GP. Sjogren's syndrome and its therapy. A therapeutic attempt using ant lymphocyte serum [Zum Sjogrensyndrom und seiner be handlung. Ein therapieversuch mit antilymphozytenglobulin] Zeitschrift Fur Laryngologie, Rhinologie, Otologie Und Ihre Grenzgebiete. 1972;51(7):429-432.

2. Alvarado Valero MC, Martínez Toldos JJ, Borras Blasco J, Almiñana Almiñana A, Pérez Ramos JM. [Treatment of persistent epithelial defects using autologous serum application]. [Tratamiento de defectos epiteliales persistentes mediante suero autologo] Arch Soc Esp Oftalmol. 2004;79(11):537-542.

3. Anderson NG, Regillo C. Ocular manifestations of graft versus host disease. Current Opinion in Ophthalmology. 2004;15(6):503507. doi: 10.1097/01.icu.0000143684.22362.46

4. Badami K, McKellar M. Allogeneic serum eye drops-a useful alternative for those unable to be autologous donors. Transfusion; Proceedings of the AABB Annual Meeting and TXPO; New Orleans (LA). 2009.

5. Bradley JC, Bradley RH, Mccartney DL, Mannis MJ. Serum growth factor analysis in dry eye syndrome. Clinical and Experimental Ophthalmology. 2008;36(8):717-720. doi: 10.1111/j.14429071.2008.01895.x.

6. Brown SM, BradleyJC. The effect of autologous serum eye drops in the treatment of severe dry eye disease: a prospective randomized casecontrol study. American Journal of Ophthalmology. 2005;140(3):565566. doi: 10.1016/j.ajo.2005.03.067

7. Chiang CC, Lin JM, Chen WL, Tsai YY. Allogeneic serum eye drops for the treatment of severe dry eye in patients with chronic graftversus-host disease. Cornea. 2007;26(7):861-863. doi: 10.1097/ ICO.0b013e3180645cd7

8. Craig JP. A natural solution to dry eye? Clinical and Experimental Ophthalmology.2008;36(2):109-110.doi:10.1111/j.14429071.2008.01708.x
9. Fuchsluger TA, Steuhl KP, Meller D. Neurotrophic keratopathy-a post-LASIK case report. 2005;222(11):901-904. doi: 10.1055/s2005-858800

10. Geerling G, Hartwig D. Autologous serum-eye-drops for ocular surface disorders. A literature review and recommendations for their application. Ophthalmologe. 2002;99(12):949-959. doi: 10.1007/s00347-002-0661-6

11. Geerling G, Maclennan S, Hartwig D. Autologous serum eye drops for ocular surface disorders. British Journal of Ophthalmology. 2004;88(11):1467-1474.

12. Geerling G, Unterlauft JD, Kasper K, Schrader S, Opitz A, Hartwig D. Autologous serum and alternative blood products for the treatment of ocular surface disorders. Ophthalmologe. 2008;105(7):623-631. doi: $10.1007 / \mathrm{s} 00347-008-1750-\mathrm{y}$

13. Harritshoj LH, Hansen MB, Julian HO. Ready-made allogenic serum eye drops for severe dry eye disease. Vox Sanguinis; Proceedings of the 21st Regional Congress of the International Society of Blood Transfusion; Lisbon, Portugal. 2011.

14. Hyon JY, Lee YJ, Yun PY. Management of ocular surface inflammation in Sjögren syndrome. Cornea. 2007;26(9 Suppl 1):S13-15. doi: 10.1097/ICO.0b013e31812f6782

15. Jaksche A, Sbeity Z, Domeier E, Fimmers R, Holz F, Loeffler KU. Undiluted versus diluted Autologous Serum Eye Drops (ASED): A prospective, randomized, double-blind study in patients with refractory dry eye-syndrome. Investigative Ophthalmology and Visual Science. 2005;46 ARVO E-abstract 2045.

16. Koffler BH. Autologous serum therapy of the ocular surface with novel delivery by platelet concentrates gel. Ocular Surface. 2006;4(4):188195. doi: 10.1016/S1542-0124(12)70165-9

17. Kojima T, Dogru M, Matsumoto Y, Goto E, Tsubota K. Tear film and ocular surface abnormalities after eyelid tattooing. Ophthal Plast Reconstr Surg. 2005;21(1):69-71.

18. Kojima T, Higuchi A, Goto E, Matsumoto Y, Dogru M, Tsubota K. Autologous serum eye drops for the treatment of dry eye diseases. Cornea. 2008;27 Suppl 1:S25-30. doi: 10.1097/ ICO.0b013e $31817 \mathrm{f} 3 \mathrm{a} 0 \mathrm{e}$ 